\title{
Towards Nanowire Tandem Junction Solar Cells on Silicon
}

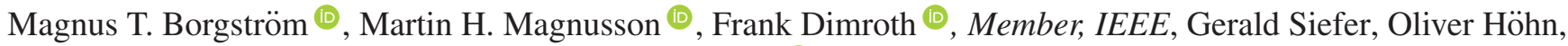 \\ Heike Riel, Senior Member, IEEE, Heinz Schmid ${ }^{\circledR}$, Member, IEEE, Stephan Wirths, Mikael Björk, \\ Ingvar Åberg, Senior Member, IEEE, Willie Peijnenburg (i), Martina Vijver, Maria Tchernycheva, Valerio Piazza, \\ and Lars Samuelson ${ }^{\circledR}$, Member, IEEE
}

\begin{abstract}
The development of photovoltaics as a serious means of producing renewable energy has accelerated greatly in the last ten years, with prices for silicon-based solar cell systems dropping dramatically in the last few years. The next great opportunity for photovoltaics following this competitiveness in prices will be to enhance the cell and panel efficiencies. It is quite generally seen that the most viable platform on which this should be realized will be as augmented silicon solar cells, in which a top cell will be combined with the silicon bottom cell in a tandem configuration, by which the efficiency can be enhanced by a factor from $20 \%$ to $50 \%$, depending on details of the approach. In this paper, we report on the status of one such approach, namely, with a top cell comprising III-V nanowires, connected to the bottom silicon cell in a two-terminal or four-terminal configuration. Among the most important opportunities, we show that a substrate-free growth, called Aerotaxy, offers a radical reduction in the total price picture. Besides the description of the key technical approaches, we also discuss the environmental issues.
\end{abstract}

Index Terms-Environmental impact, nanowires (NWs), photovoltaics (PVs), tandem cells.

Manuscript received October 31, 2017; revised December 22, 2017 and February 20, 2018; accepted March 6, 2018. Date of publication April 16, 2018; date of current version April 19, 2018. This work was supported in part by the Swedish Energy Agency, in part by the Swedish Research Council, in part by the Knut and Alice Wallenberg Foundation, in part by Nanolund, in part by Myfab, and in part by the European Union's Horizon 2020 research and innovation programme under Grant 641023 (Nano-Tandem). (Corresponding author: Lars Saтuelson.)

M. T. Borgström, M. H. Magnusson, and L. Samuelson are with Solid State Physics/NanoLund, Lund University, Lund 22100, Sweden (e-mail: magnus.borgstrom@ftf.lth.se; martin.magnusson@ftf.lth.se; lars.samuelson@ ftf.lth.se).

F. Dimroth, G. Siefer, and O. Höhn are with the Fraunhofer Institute for Solar Energy Research, Freiburg 79115, Germany (e-mail: frank. dimroth@ise.fraunhofer.de; gerald.siefer@ise.fraunhofer.de; oliver.hoehn@ fraunhofer.de).

H. Riel, H. Schmid, and S. Wirths are with IBM Research Laboratory, Ruschlikon CH-8803, Switzerland (e-mail: hei@zurich.ibm.com; sih@zurich.ibm.com; ZRLWIR@ch.ibm.com).

M. Björk and I. Åberg are with Sol Voltaics AB, Lund 223 63, Sweden (e-mail: mikael.bjork@ solvoltaics.se; ingvar.aberg@ solvoltaics.se).

W. Peijnenburg and M. Vijver are with the University of Leiden, Leiden 2311 EZ, The Netherlands (e-mail: Peijnenburg@cml.leidenuniv.nl; vijver@ cml.leidenuniv.nl).

M. Tchernycheva and V. Piazza are with the C2N-CNRS, University Paris Sud, University Paris Saclay, Orsay 91044, France (e-mail: maria.tchernycheva@u-psud.fr; valerio.piazza@u-psud.fr).

Color versions of one or more of the figures in this paper are available online at $\mathrm{http}: / /$ ieeexplore.ieee.org.

Digital Object Identifier 10.1109/JPHOTOV.2018.2816264

\section{INTRODUCTION}

$\mathbf{S}$ ILICON (Si)-based photovoltaic (PV) cells are the dominant technology for terrestrial solar energy conversion. After many decades of research and development, efficiencies have essentially saturated with the best devices measuring $26.7 \%$ in the laboratory [1], [2]. Significantly higher conversion efficiencies up to $38.8 \%$ under one sun illumination have been reached with multijunction cells based on III-V semiconductors in flat plate configuration [3]. However, this technology has been too expensive for use in large area flat-plate modules, and the combination of high-efficiency solar cells in tandem configuration on a Si platform has recently attracted great attention using thin film III-V materials [4] as well as perovskites [5]. The use of III-V nanowires (NWs) allows a significant reduction in material needs and has the potential to exceed the performance of conventional devices after optimizing synthesis, and processing for materials quality, surface passivation, and minimizing contact resistance. NWs offer to combine lattice mismatched materials with band gaps optimized for absorption of the solar spectrum due to the reduction in lattice matching requirements making it possible to grow them monolithically on Si [6], [7]. The efficiency of NW-based PVs is rapidly increasing [8], and references therein, with recent record efficiencies of $13.8 \%$ for vapor liquid solid (VLS) [9] grown indium phosphide (InP) NWs [10] and 15.3\% efficiency for VLS grown gallium arsenide (GaAs) NWs [11]. The current record in NW PV efficiency is $17.8 \%$, reported for top down etched InP NWs [12]. III-V NWs in combination with today's Si PV technology have the potential of simultaneously allowing very high performance, efficient use of materials, and low cost. The first step to multijunction technology has been taken where InP NW PV has been integrated into Si [13]. More importantly, open-circuit voltage addition of GaAs NW PV grown on a Si solar cell, previously modeled [14], was recently shown [15]. For improved performance, the NW materials band gap has to be tuned for an optimal absorption of the solar spectra in combination with Si. This involves the use of ternary NW materials like (doped) gallium indium phosphide (GaInP) and gallium arsenide phosphide (GaAsP), which represent the high band gap segment in a tandem junction geometry. In this contribution, we report on the concepts and most recent advances made with respect to integrating NWs into a $\mathrm{Si}$ solar cell platform. Together we pursue three main concepts for 


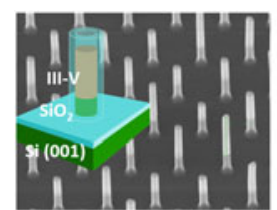

(a)

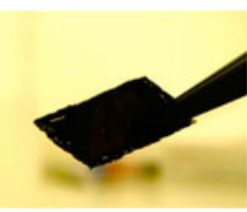

(b)

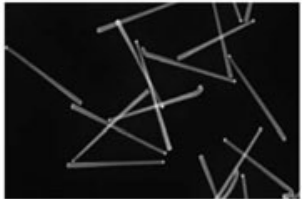

(c)
Fig. 1. Simple sketch of the three different concepts. (a) Schematic of template-assisted epitaxy (TASE) and SEM image of a fabricated structure, (b) SEM image of epitaxially grown NWs, peeled off and held with a tweezer, intended for transfer to a Si cell. The membrane is black due to efficient light absorption in the NW membrane, (c) substrate-free growth by Aerotaxy, example of NWs grown by Aerotaxy.

increasing the efficiency of Si-based PV by integration with NWs and evaluate the environmental performance of using NWs instead of thin films by means of a life-cycle assessment (LCA) approach in which different impact categories as well as toxicity are considered.

\section{NANO-TANdem Cell Concepts (SEe Fig. 1)}

\section{A. Concept I: Direct Growth of NWs on a Silicon Solar Cell}

In this approach, we use a catalyst-free growth technique to directly integrate III-V semiconducting NWs into Si using selective area epitaxy within nanotube templates. The benefit of this template-assisted selective epitaxy (TASE) growth mode is the ability to precisely control the crystal morphology and location of the III-V NWs, as well as to directly grow III-Vs on many Si crystalline orientations, even poly-Si [16]. TASE, hence, enables the growth of vertical NWs on standard (001) Si and thus the use of cost-efficient substrates. Furthermore, TASE offers an attractive way to establish epitaxial contact with $\mathrm{Si}$ at the NW/Si interface and its use as a tunnel junction [17]. The use of metal organic vapor phase epitaxy (MOVPE), which is a mature technique and direct growth on $\mathrm{Si}$, allows large area deposition, which can be facilitated by holographic methods or nano-imprint lithography for patterning.

In this approach, the Si bottom solar cell uses a planar pnjunction with a polished front surface. Therefore, light trapping structures for increasing absorption may only be integrated into the back of the Si substrate.

\section{B. Concept II: NWs Synthesized on InP or GaAs Substrate for Peel Off, Transfer, and Substrate Reuse}

The benefit of this approach is that NW nucleation on a native substrate is the most controlled, and the use of mature MOVPE. Here, the NWs are embedded in a polymer film after growth, peeled off from the substrate, and bonded to Si. Then, the III-V substrate can be reused for further growth. The process of peeling off and transferring micro-NWs has successfully been shown by the Atwater group [18], [19]. The challenge is to develop the method for NWs with dimensions and frictional properties much smaller than those for micrometer diameter NWs. Economically viable prepatterning, in this case with metal nanoparticles as a catalyst for the NW growth, is essential. In concept I, the two cells are monolithically integrated, and therefore by necessity

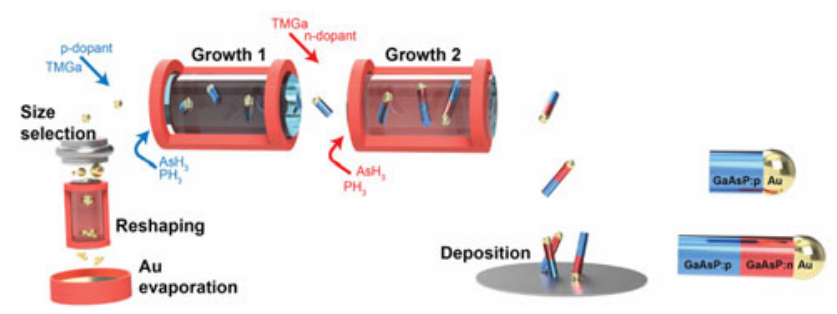

Fig. 2. Schematic of the Aerotaxy process, where Au seed particles are grown into $\mathrm{GaAs}(\mathrm{P}) \mathrm{NW}$ s in a continuous flow. The sequential growth of the intended NW device design is shown in the enlarged model NWs to the right.

current matched, which creates performance issues since the color content in the sunlight varies as a function of time of the day and year. In concept II, the option is either to integrate the NW array by forming a direct semiconductor-semiconductor contact, or to form a (semi) transparent contact on the bottom side of the NW-containing membrane. The second case allows the top and bottom cells to operate individually with a total of four leads out which results in an advantage not requiring current matching. In this case, light-trapping structures can be integrated into the Si front surface between the NW film and the Si bottom cell. This helps to enhance the light propagation and therefore absorption in the Si bottom cell.

\section{Concept III: Substrate-Less NW Growth: Aerotaxy}

Aerotaxy was invented partly to test the fundamentals of NW growth in the absence of a substrate and to address the high cost of producing NWs for large-area applications, especially solar cells. In Aerotaxy, NWs grow from seed particles directly in the gas phase, and the resulting NW aerosol can be transferred into an ink for storage and further processing. One remarkable aspect of Aerotaxy is that this continuous process results in growth rates on the order of $1 \mu \mathrm{m} / \mathrm{s}$, which is $100-1000$ times faster than MOVPE growth [20]. Fig. 2 shows an illustration of the Aerotaxy process, for the case of GaAsP pn-junction NWs. The experimental, research scale, Aerotaxy equipment in use at Lund University yields approximately $2 \mathrm{mg}$ of size-selected NWs per hour, which is equivalent to $4 \mathrm{NWs}$ per $\mu \mathrm{m}^{2}$ on a $4^{\prime \prime}$ wafer. Note that Aerotaxy needs no lithography and is inherently scalable. Similar to concept II, this approach allows a choice of current matching or not.

\section{Concept I Results}

In this approach, a high-energy band gap III-V NW-Si tandem junction solar cell is targeted by epitaxial growth of the III-V NW junction using TASE on the top of the Si planar cell. In order to achieve a high-energy band gap absorption complementing that of $\mathrm{Si}$, a ternary III-V NW with a band gap of approximately $1.7 \mathrm{eV}$ is required. Therefore, we investigated GaAsP and InGaP directly grown on $\mathrm{Si}$. The vertical oxide templates were fabricated by spin-coating a sacrificial carbon layer (1.5 $\mu \mathrm{m}$ thick) on the substrate, which was covered by a silicon dioxide $\left(\mathrm{SiO}_{2}\right)$ layer and patterned by lithography (e.g., electron beam lithography or interference lithography, for cost reasons) and reactive ion etching. We used the patterned $\mathrm{SiO}_{2}$ layer as 


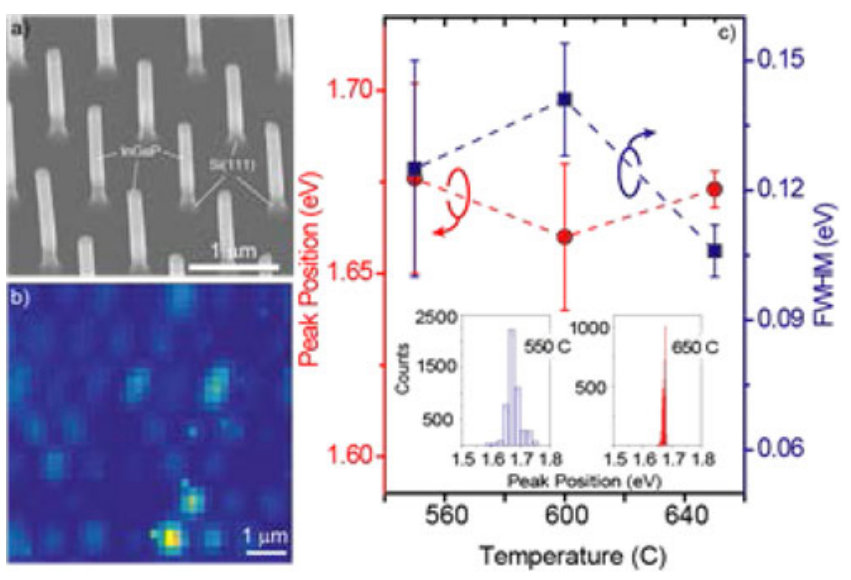

Fig. 3. (a) Tilted view SEM micrograph of an InGaP NW array grown on $\mathrm{Si}(111)$; the oxide template is stripped. (b) PL intensity map of an NW array grown at $550^{\circ} \mathrm{C}$. (c) PL peak positions and FWHM for InGaP NWs grown at $550{ }^{\circ} \mathrm{C}, 600^{\circ} \mathrm{C}$, and $650{ }^{\circ} \mathrm{C}$. PL peaks and FWHM were extracted from spectral maps covering NW arrays. The step size between PL spectra was $100-200 \mathrm{~nm}$. Insets show the temperature-dependent peak distribution.

a hard mask to define sacrificial carbon NWs with diameters between 100 and $200 \mathrm{~nm}$ by dry etching. To create the vertical oxide tube, we deposited $\mathrm{SiO}_{2}$ homogeneously around the NWs, after which the top part of the shell was opened and the sacrificial carbon NW was removed by etching. This process is suitable for large area growth and any Si substrate orientation. Furthermore, the thermal budget of the developed process including NW growth allows for the implementation with Si PV cell fabrication.

The selective epitaxy of GaAsP and InGaP on $\mathrm{Si}(001)$ and $\mathrm{Si}(111)$ within vertical templates was explored at growth temperatures between $550{ }^{\circ} \mathrm{C}$ and $650{ }^{\circ} \mathrm{C}$ and the influence of the V/III ratio (20-120) was studied. Higher growth rates were observed for $\mathrm{InGaP}$ as compared to GaAsP. Using $\mathrm{Si}(001)$, the composition of GaAsP as well as InGaP was nonuniform under the process conditions evaluated. This was revealed by cathodoluminescence (CL) and electron dispersive x-ray (EDX) measurements using NW arrays as well as individual NWs. We ascribe the inhomogeneity to different Ga versus In and As versus $\mathrm{P}$ incorporation for $\mathrm{InGaP}$ and GaAsP, respectively, on the growth facets. A previous study showed the formation of (111)B and $\{110\}$ growth interfaces on $\mathrm{Si}(100)$ [21]. This is different in the case of $\mathrm{Si}(111)$ where only a nearly atomically flat, a horizontal (111)B top facet is present. We, therefore, focused on $\mathrm{Si}(111)$ substrates, as illustrated in Fig. 3, showing an $\mathrm{X}$ transmission electron micrographs (X-TEM) image of an InGaP NW with a single (111)B facet. Fig. 3 shows room temperature photoluminescence (PL) data of an NW array, with a PL area intensity map in Fig. 3(b). We attribute the spots with higher intensities to nonuniform filling of the templates leading to parasitic growth outside the template. The PL of an NW array $\left(7 \times 7 \mu \mathrm{m}^{2}\right)$ comprising approximately $50 \mathrm{NWs}$ was mapped using an excitation wavelength of $532 \mathrm{~nm}$ and a step size of $100-200 \mathrm{~nm}$. The NWs within the arrays exhibit lengths of up to $1.5 \mu \mathrm{m}$, widths of $150 \mathrm{~nm}$ in a pitch of $1 \mu \mathrm{m}$ and were grown at a constant $\mathrm{V} / \mathrm{III}$ and group III gas ratio [TMGa/(TMGa + TMIn), with $\mathrm{TMGa}=$ trimethylgallium and $\mathrm{TMIn}=$ trimethylindium] of

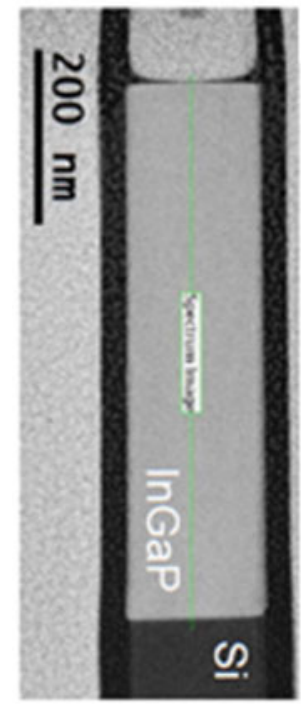

(a)

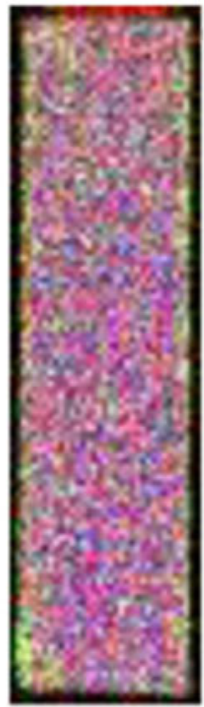

(b)

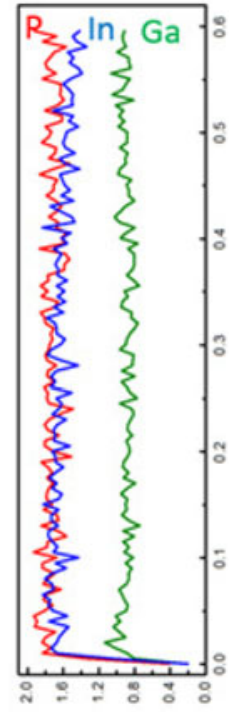

(c)
Fig. 4. TEM and EDX measurements of a single $\mathrm{InGaP}$ NW grown in a template on Si(111), showing a uniform NW geometry with a flat interface to Si and flat top facet and a nearly homogeneous composition along the NW.

26 and 0.43 , respectively. The highest growth temperature lead to the most favorable optical properties, like narrow full width at half maximum (FWHM) and PL peak distribution in the array, as summarized in Fig. 3(c). The peak position of $1.67 \mathrm{eV}$ (approx. $30 \% \mathrm{Ga}$ ) is close to the required value for efficient tandem solar cells.

Fig. 4 shows the cross-section of a representative InGaP NW enclosed in the template with the corresponding EDX analysis. A homogeneous in-plane composition is observed with a slight decrease of In content along the growth direction, which can be attributed to the difference in surface diffusion length between In and Ga species, on the oxide mask. This phenomenon is known in selective area epitaxy where the species fluxes are modified in the proximity of the masking area. The TEM show a flat NW growth surface and a zinc blende (ZB) crystal with planar defects on (111) direction and misfit dislocations located at the NW-Si interface. These experiments suggest that $\mathrm{InGaP}$ with the proper composition can be integrated directly into $\mathrm{Si}$ using TASE.

Nanoscale analyses are essential to probe individual NW pnjunctions in order to analyze the material quality, to assess the NW-to-NW homogeneity, and to detect eventual failures. CL and electron beam induced current (EBIC) microscopy techniques allow to probe optical properties and current generation with a resolution of several tens of nanometers.

We performed EBIC microscopy and single NW currentvoltage characterization on TASE grown InGaP NWs containing a pn-homojunction by introducing diethylzinc (DEZn) and disilane $\left(\mathrm{Si}_{2} \mathrm{H}_{6}\right)$, respectively. As expected, we observe a strong induced current at the position of the pn-junction (see Fig. 5). An important step will be to demonstrate an InGaP-Si tunnel junction. Initial results using a highly n-doped InGaP NW on degenerately p-doped $\mathrm{Si}$ show the appearance of a negative differential resistance but only after cooling to $150 \mathrm{~K}$. While 


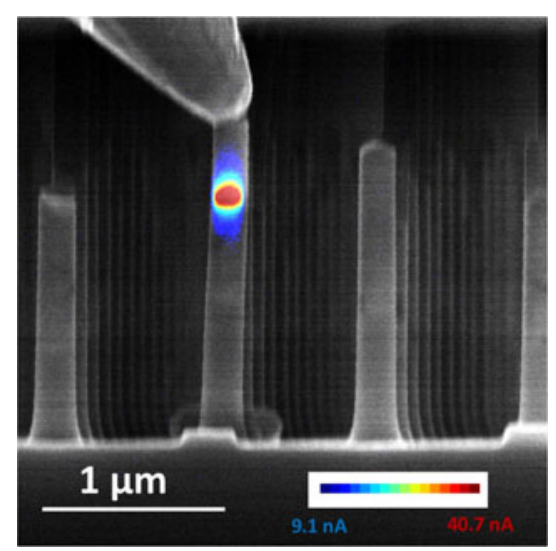

Fig. 5. Electrical probe contacting an individual InGaP NW grown on Si. Under reverse bias, a generated EBIC current reveals the position of the InGaP pn-junction.

promising, this clearly demands continued work to improve the tunnel junction. Eventually, surface passivation can be further improved by stripping the template oxide from the NWs and growing a higher bandgap III-V shell around the NWs.

\section{E. Concept II Results}

In a first step to realize high energy band gap NWs for NW Si tandem junction solar cells by MOVPE, peel off, and transfer, we synthesized and characterized p-doped ternary GaInP NWs aiming for a band gap of about $1.7 \mathrm{eV}$ in a pattern defined by nano-imprint lithography for optimal light absorption in InP NWs [22]. InGaP was chosen over GaAsP due to previous knowledge of doping. It was found that the use of DEZn for p-type doping leads to more efficient TMGa pyrolysis, which increases the Ga content in the NWs as a function of attempted doping [23]. The opto-electrical properties of ternary InGaP NWs grown by Au-assisted MOVPE were characterized by CL and EBIC. CL showed that the NWs had a band gap close to $1.7 \mathrm{eV}$ and a good NW-to-NW and intra-NW compositional homogeneity. In two analyzed samples, the room-temperature peak emission and its deviations were $1.739 \pm 0.041 \mathrm{eV}$ and $1.640 \pm$ $0.022 \mathrm{eV}$, respectively. For both samples, the variation of the peak CL energy along the NW axis was less than $20 \mathrm{meV}$. EBIC mapping allowed to directly visualize the internal field region having a width varying from $500 \mathrm{~nm}$ to $1 \mu \mathrm{m}$. Diffusion lengths of minority carriers around 150-200 nm were determined by analyzing the EBIC profiles.

\section{F. NW Growth, Peel Off, and Reuse of Substrate}

In order to make epitaxially grown NW solar cells economically viable, one requirement within the project is to make technology for recycling of the substrate available, diminishing substrate costs. In Fig. 6, scanning electron microscope (SEM) images are shown of 1) selective area grown NWs grown on an InP substrate, 2) the sample surface after the first peel off, and 3) NWs grown after recycling the substrate twice, i.e., using the sample for growth three times. The NWs are indistinguishable, indicating that further recycling of the substrate is feasible.

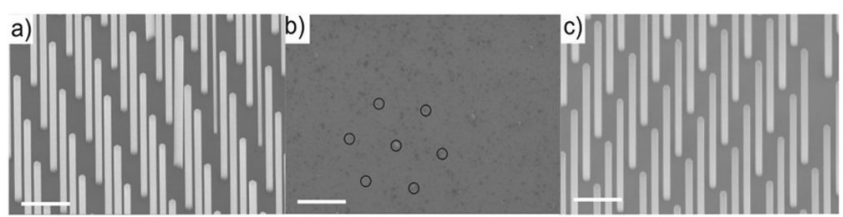

Fig. 6. NW growth and peel off. SEM images of (a) selective area MOVPE grown NWs. First time of substrate use. (b) Substrate after first peel off. A hexagon of circular markers indicates the position of openings in the SiN growth mask for clarity. (c) Selective area MOVPE grown NWs, third time of substrate use. The scale bar for all images is $1 \mu \mathrm{m}$. (a) and (c) are taken under a $30^{\circ}$ angle toward the normal of the plane. (b) shows a top view image.

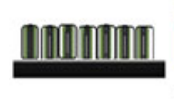

(a)

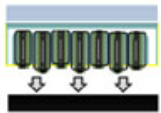

(b)

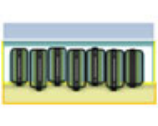

(c)

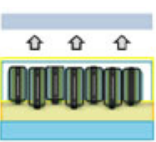

(d)

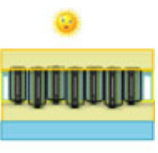

(e)
Fig. 7. Process flow for integrating peeled off NWs into cells, ready to stack on the Si cell according to concept II. After NW growth (a), the NWs are embedded in a polymer and transferred to a carrier substrate (e.g., UV-release/PET tape) by peeling (b). The rear transparent conductor is added (c) and after bonding to a glass substrate, the carrier is released (d) prior to the deposition of the front transparent conductor.

Regarding the quality of the NWs regrown on the used substrates, we identify the NW crystal as wurtzite. From the SEM images in Fig. 6(a) and (c), it can be seen that the NWs are self-similar with respect to morphology. We evaluated the optical quality by PL spectroscopy and observed characteristic behavior that can be explained by differences in twinning sequence leading to varying lengths of wurtzite and ZB segments in the NWs [24], [25], independent of the number of times the substrate had been used.

\section{G. Cell Integration of Peeled Off Membranes}

While substrate reuse was demonstrated using selective area grown NWs, the highest efficiency of epitaxially grown NWs has been shown by GaAs or InP NWs grown by the VLS method. Therefore, such NWs were peeled and integrated according to the process flow shown in Fig. 7.

The mechanical properties of the NW-polymer-carrier system determine the ability to peel the NWs. For integration, we grew GaAs NWs on a $2^{\prime \prime}$ GaAs wafers using a square pattern with $500 \mathrm{~nm}$ pitch. One important parameter is the diameter of the $\mathrm{NW}$, which together with the pitch is set by the requirements for optical absorption, but also by the need for additional radial passivation layers [11]. To enable peeling, we had to limit the radial passivation layer thickness to $15 \mathrm{~nm}$ on a $\sim 165 \mathrm{~nm}$ diameter core, less than the ideal $30-40 \mathrm{~nm}$ required for ideal passivation [11]. The current voltage curve of a $5 \times 5 \mathrm{~mm}^{2}$ peeled NW GaAs cell with the efficiency of $3.5 \%$ is shown in Fig. 8. The short-circuit current density $\left(J_{\mathrm{sc}}=6.8 \mathrm{~mA} / \mathrm{cm}^{2}\right)$ is lower than on the best GaAs NW cells made from epitaxially grown NWs $\left(J_{\mathrm{sc}}>20 \mathrm{~mA} / \mathrm{cm}^{2}\right)$. In addition to the thinner than the ideal passivation layer, this can be attributed to losses from the $\mathrm{Au}$ catalyst seed particle not being removed [26]. For comparison, sister wafers with cells integrated into wafer resulted in $J_{\mathrm{sc}}$ 


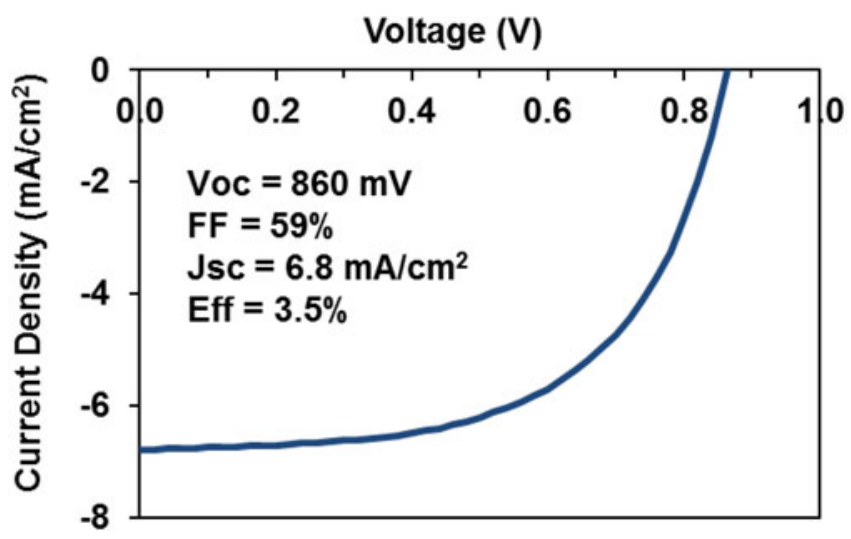

Fig. 8. Current-voltage curve of a peeled GaAs NW cell. The test conditions are close to 1 sun, $\mathrm{AM} 1.5 \mathrm{G}$, and measured at room temperature; however, the measurement was not calibrated.

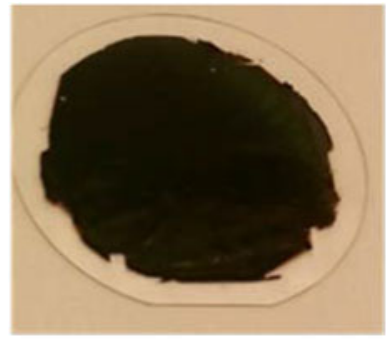

(a)

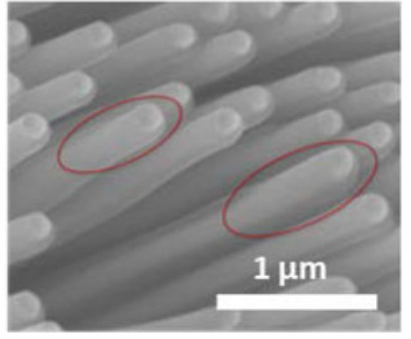

(b)
Fig. 9. (a) A peeled GaAs NW membrane, after transfer to a $2^{\prime \prime}$ glass carrier. (b) We found that NWs on these membranes are tilted. Subsequent dry etching damages the side-wall of the emitter and pn-junction, causing shunt leakage in many cells. Specifically, the oxide near the top of the NWs is fully etched (see circles). We can avoid this issue by combining the NWs and cell integration concepts from concept II with the membrane procedure of concept III.

of $7 \mathrm{~mA} / \mathrm{cm}^{2}$ (with $15 \mathrm{~nm}$ passivation) and $J_{\mathrm{sc}}$ of $12 \mathrm{~mA} / \mathrm{cm}^{2}$ (with $40 \mathrm{~nm}$ passivation). Both of these wafers were integrated with the Au seed particle remaining. This shows that the peeling process and removing the native wafer substrate are not the reason for the low current. The open-circuit voltage was nearly identical to the open-circuit voltage of cells integrated into wafer, while the fill factor was significantly lower, a result of series resistance at least in part due to the not yet optimized rear contact.

Note that the high open-circuit voltage observed in this cell was higher than typical. Most cells under test on several peeled membranes were heavily shunted. We determined that despite the appealing visual appearance of the peeled membranes, as shown in Fig. 9(a), the NWs in the membranes were tilted by approximately $45^{\circ}$, causing a narrow process window for the emitter contact etch, causing shunting of the pn-junction located near the top of the NW [see Fig. 9(b)]. While the exact reason for the NW tilt is not clear, an investigation showed that such tilt is present already before peeling after the polymer dried out. One plausible explanation is that evaporation of solvents from the polymer may have resulted in stress, tilting the NWs. To address the shortcomings of the peeling method, we will instead use the membrane formation process developed for substrateless Aerotaxy grown NWs (concept III) also for MOVPE grown
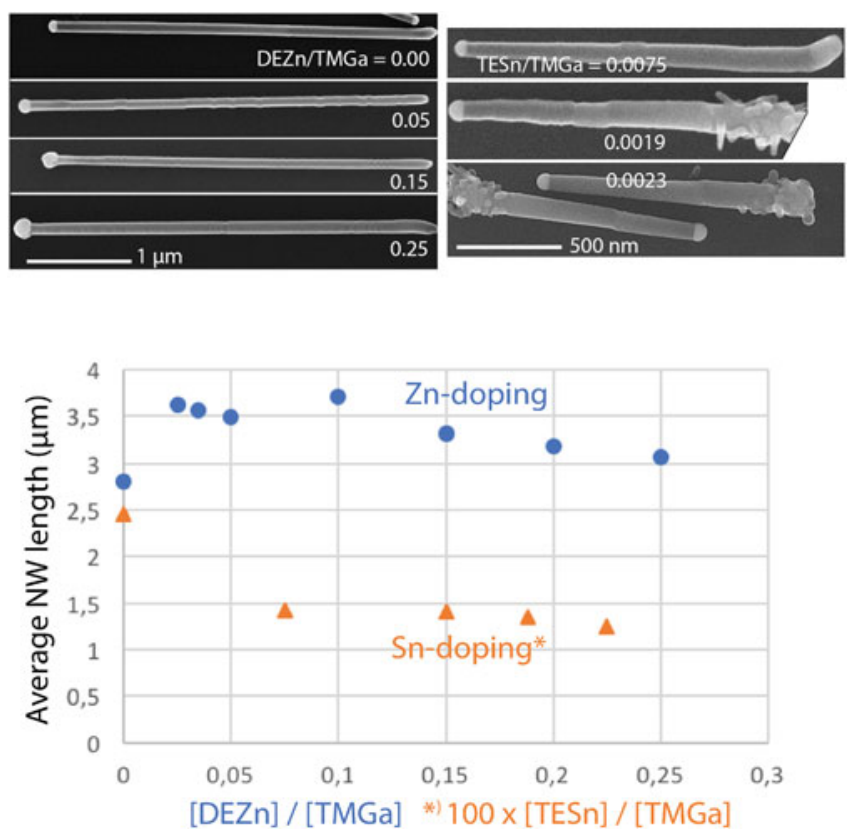

Fig. 10. Top: SEM images of typical Aerotaxy NWs after Zn and Sn doping, where the dopant is introduced together with the TMGa. Bottom: Influence of dopants on the NW length. Note that the TESn concentration is $100 \times$ less than that of DEZn.
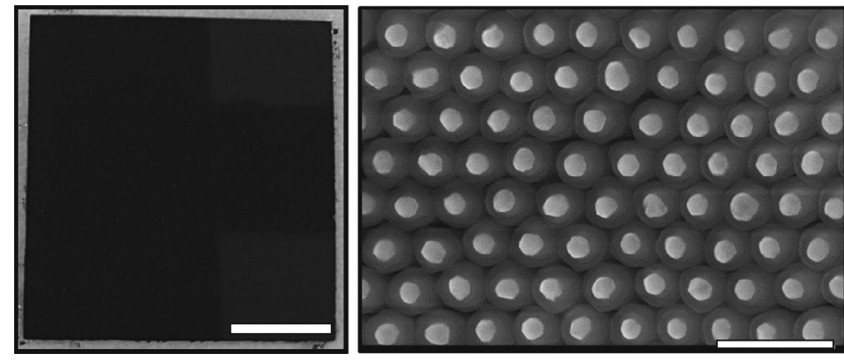

Fig. 11. Left: $30 \times 30 \mathrm{~mm}$ GaAs NW membrane made from a colloidal solution, bonded to a glass carrier substrate. The scale bar is $10 \mathrm{~mm}$. Right: Close-up view of aligned NWs in the membrane. The scale bar is $1 \mu \mathrm{m}$.

NWs, sonicated from the wafer substrate. As discussed further below, we have shown that GaAs NWs having optimized radial passivation layers can be nearly perfectly aligned without tilt on a centimeter scale (see Fig. 11). The rest of the integration process remains the same, as per Fig. 7. Thus, there is a clear path to address the issues encountered in the initial cells fabricated.

\section{H. Concept III Results}

Aerotaxy can produce NWs of excellent crystalline and optical quality, and we have so far demonstrated the following materials and dopants: pure GaAs [20], p-GaAs (with Zn) [27], n-GaAs (with Sn) [28], pn-GaAs (Zn and Sn) [29], and undoped $\mathrm{GaAs}_{1-\mathrm{x}} \mathrm{P}_{\mathrm{x}}$ in the range $0<x<42 \%$ [30], which is why we chose GaAsP rather than GaInP for Aerotaxy; the ideal composition for a tandem diode on $\mathrm{Si}$ is approximately $\mathrm{GaAs}_{0.75} \mathrm{P}_{0.25}$. $\mathrm{Zn}$ and $\mathrm{Sn}$ doping behave quite differently, as illustrated in Fig. 10. While DEZn does not have a detrimental effect on the NW growth rate, even a small amount of TESn reduces the NW 
length measured after growth. It is also clear from the SEM images that the use of TESn as a dopant precursor can give rise to parasitic radial growth, especially at the base end of the NW. For this reason, we are planning to evaluate n-type doping by use of dihydrogensulfide $\left(\mathrm{H}_{2} \mathrm{~S}\right)$ as a dopant precursor. For Aerotaxy using DEZn as a p-type dopant source, we note that the seed particle diameter observed after growth increases with dopant molar fraction used. The NW diameter does not show a corresponding increase, which most probably is a consequence of the expected changes in a wetting angle due to the presence of DEZn [31], [32]. PL and electrical characterization confirm that the doping with $\mathrm{Zn}(\mathrm{Sn})$ results in $\mathrm{p}(\mathrm{n})$-type NWs with carrier concentrations around $1-5 \times 10^{19} \mathrm{~cm}^{-3}$.

Aerotaxy is a promising route to low-cost GaAs NW material fabrication for large-scale solar applications. However, with this approach, the control of NW position on a substrate is lost as the NWs are grown continuously and directly in the gas phase, forming a dust of randomly oriented NWs. Sol Voltaics has developed a proprietary technology to align randomly distributed NWs, collected in a liquid solvent, into a thin membrane of ordered, vertically aligned, and oriented NWs.

Fig. 11 shows a $30 \times 30 \mathrm{~mm}^{2}$ GaAs NW membrane bonded to a glass substrate; to illustrate that this method not only applies to Aerotaxy-grown NWs, these NWs are radially passivated and grown by use of MOVPE. The average alignment and orientation yield over the membrane is $98.9 \%$ and $99.4 \%$, respectively, with a surface density of $4.6 \mathrm{NWs}$ per $\mu \mathrm{m}^{2}$ demonstrating the technology.

\section{Environmental Impacts}

We assessed the environmental impacts of the three basic concepts developed for integrating NWs into a Si solar cell platform by performing a preliminary LCA. The LCA is based on the evaluation of the environmental impacts from cradle-togate: production of raw materials, use, disposal of materials, and energy requirements to manufacture the technology in the laboratory. The laboratory technology is the basis for the development of scenarios and the development of a sustainability roadmap for the up-scaled commercial production of NW-based tandem solar cells. The functional unit of lab-scale production of one 4-in NW film on a Si wafer was selected as the basis for the LCA. The system boundaries include the laboratory processes and the related material and energy inputs for production of one wafer for the different manufacturing routes. We quantified the system of processes for the different manufacturing routes and the related material and energy data, and used the EcoInvent database (version 2.2) to compile data for the life cycle inventory of the background system. The CMLCA software developed at Leiden University was used to compile the system of processes for the different manufacturing routes, the related material and energy inputs, and the actual impact assessments.

The initial LCA showed that carbon emissions induce the majority of the environmental impacts. Carbon emissions for the production of one 4-in Si wafer at the laboratory scale equal $140 \mathrm{~kg} \mathrm{CO}_{2}$-eq, which is equal to $54250 \mathrm{~kg} \mathrm{CO}_{2}$-eq for the labscale production of $1 \mathrm{~m}^{2}$ of the solar cell surface. Fig. 12 shows a breakdown of the carbon emissions per process for concept II.

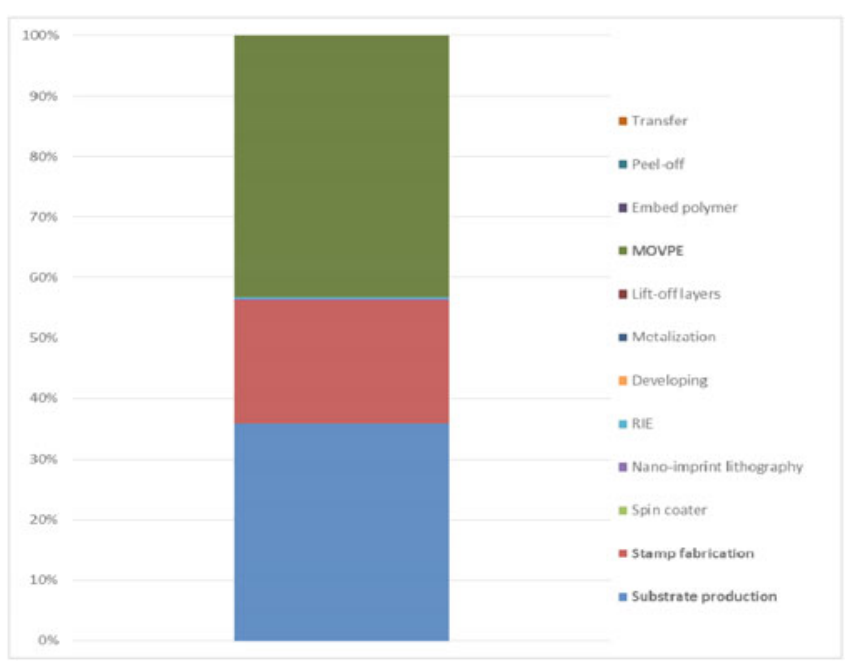

Fig. 12. Carbon emissions contribution per process for concept II (no substrate reuse, no stamp reuse), RIE = reactive ion etching.

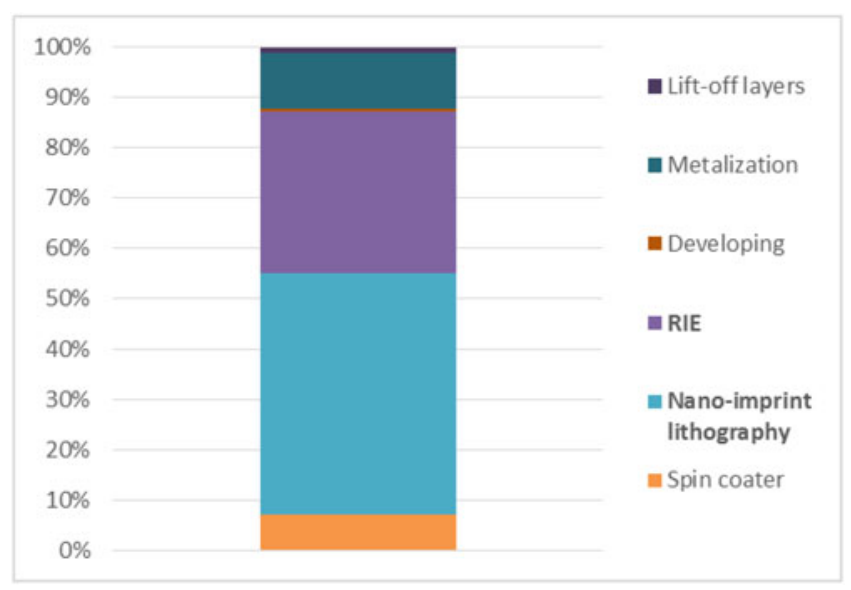

Fig. 13. Carbon emissions contributions per process upstream in the value chain for concept II.

As can be seen from Fig. 12, production of the III-V substrate, fabrication of the stamp, and MOVPE processes have the highest impacts in terms of carbon emissions. As substrate and wafer production make up more than $50 \%$ of the total carbon emissions, reuse of the substrate and stamp will have an important impact in reducing carbon emissions.

Contribution analysis allows investigating which processes up-stream in the value chain contribute most to carbon emissions. Fig. 13 shows that mainly nano-imprint lithography and reactive ion etching contribute to carbon emissions.

The preliminary LCA for the laboratory production of one 4-in NW Si wafer shows that it is mainly the electricity requirements of the processes that contribute to carbon emissions. No more than $5 \%$ of the carbon emissions are due to disposal of solvent mixtures and material usage has a very small impact. Given likely options for reuse of materials and reduction of energy input, up-scaling of the concepts toward commercial production of the solar cell technology will most likely induce a significant reduction of the environmental impacts. 


\section{CONCLUSION}

Based on the research presented here, NWs offer a highly interesting opportunity in augmenting the performance of standard Si PV cells by adding a layer of vertically oriented III-V NWs on the top of the Si cell. In such a tandem cell configuration, NWs with inherently efficient light absorption can potentially enhance the Si-only cell efficiency by more than $20 \%$ relative, as shown for thin films [1].

\section{ACKNOWLEDGMENT}

The authors would like to thank Dr. E. Barrigón, G. Otnes, V. Dagyte, X. Zeng, Dr. N. Anttu, Prof. M.-E. Pistol, Y. Cheng, I. Geiselaars, G. Pallas, Dr. W. Metaferia, S. Sivakumar, Dr. M. Heurlin, L. Johansson, G. Vescovi, Dr. D. Lindgren, C. Sundvall, D. Asoli, Dr. J. Castillo-León, N. Bologna, M. Knoedler, J. Benick, J. C. Goldschmidt, and H. Hauser for helpful collaboration within the Nano-Tandem project.

This publication reflects only the authors' views and the funding agency is not responsible for any use that may be made of the information it contains.

\section{REFERENCES}

[1] M. A. Green et al., "Solar cell efficiency tables (version 50)," Prog. Photovolt., vol. 25, no. 7, pp. 668-676, 2017.

[2] K. Masuko et al., "Achievement of more than $25 \%$ conversion efficiency with crystalline silicon heterojunction solar cell," IEEE J. Photovolt., vol. 4, no. 6, pp. 1433-1435, Nov. 2014.

[3] M. A. Green and S. P. Bremner, "Energy conversion approaches and materials for high-efficiency photovoltaics," Nature Mater. Rev., vol. 16, no. 1, pp. 23-34, 2017.

[4] S. Essig et al., "Realization of $\mathrm{GaInP/Si}$ dual-junction solar cells with 29.8\% 1-sun efficiency," IEEE J. Photovolt., vol. 6, no. 4, pp. 1012-1019, Jul. 2016.

[5] K. A. Bush et al., "23.6\%-efficient monolithic perovskite/silicon tandem solar cells with improved stability," Nature Energy, vol. 2, 2017, Art. no. 17009.

[6] T. Mårtensson et al., "Epitaxial III-V NWs on silicon," Nano Lett., vol. 4, no. 10, pp. 1987-1990, 2004.

[7] E. P. A. M. Bakkers, M. T. Borgström, and M. A. Verheijen, "Epitaxial growth of III-V NWs on group IV substrates," MRS Bull., vol. 32, no. 2, pp. 117-122, 2007.

[8] G. Otnes and M. T. Borgstrom, "Towards high efficiency NW solar cells," Nano Today, vol. 12, pp. 31-45, 2017.

[9] R. S. Wagner and W. C. Ellis, "Vapor-liquid-solid mechanism of single crystal growth," Appl. Phys. Lett., vol. 4, no. 5, pp. 89-90, 1964.

[10] J. Wallentin et al., "InP NW array solar cells achieving 13.8\% efficiency by exceeding the ray optics limit," Science, vol. 339, no. 6123, pp. 10571060, 2013.

[11] I. Aberg et al., "A GaAs NW array solar cell with $15.3 \%$ efficiency at 1 sun," IEEE J. Photovolt., vol. 6, no. 1, pp. 185-190, Jan. 2016.

[12] D. van Dam et al., "High-efficiency NW solar cells with omnidirectionally enhanced absorption due to self-aligned indium-tin-oxide Mie scatterers," ACS Nano, vol. 10, no. 12, pp. 11414-11419, 2016.

[13] M. Heurlin et al., "Axial InP NW tandem junction grown on a silicon substrate," Nano Lett., vol. 11, no. 5, pp. 2028-2031, 2011.

[14] Y. Hu, M. Li, J. J. He, and R. R. LaPierre, "Current matching and efficiency optimization in a two-junction NW-on-silicon solar cell," Nanotechnology, vol. 24, no. 6, 2013, Art. no. 065402.

[15] M. Q. Yao et al., "Tandem solar cells using GaAs NWs on Si: Design, fabrication, and observation of voltage addition," Nano Lett., vol. 15, no. 11 , pp. 7217-7224, 2015.

[16] H. Schmid et al., "Template-assisted selective epitaxy of III-V nanoscale devices for co-planar heterogeneous integration with Si," Appl. Phys. Lett., vol. 106, no. 23, 2015, Art. no. 233101.

[17] M. T. Bjork et al., "Si-InAs heterojunction Esaki tunnel diodes with high current densities," Appl. Phys. Lett., vol. 97, no. 16, 2010, Art. no. 163501.
[18] A. C. Tamboli et al., "Wafer-scale growth of silicon microwire arrays for photovoltaics and solar fuel generation," IEEE J. Photovolt., vol. 2, no. 3, pp. 294-297, Jul. 2012.

[19] M. D. Kelzenberg et al., "Enhanced absorption and carrier collection in Si wire arrays for photovoltaic applications," Nature Mater., vol. 9, no. 3, pp. 239-244, 2010.

[20] M. Heurlin et al., "Continuous gas-phase synthesis of NWs with tunable properties," Nature, vol. 492, no. 7427, pp. 90-94, 2012.

[21] M. Borg et al., "Vertical III-V NW device integration on Si(100)," Nano Lett., vol. 14, no. 4, pp. 1914-1920, 2014

[22] G. Otnes et al., "Strategies to obtain pattern fidelity in NW growth from large-area surfaces patterned using nanoimprint lithography," Nano Res., vol. 9, no. 10, pp. 2852-2861, 2016.

[23] G. Otnes, M. Heurlin, X. L. Zeng, and M. T. Borgstrom, " $\operatorname{In}_{\mathrm{X}} \mathrm{Ga}_{1-\mathrm{x}} \mathrm{P}$ NW growth dynamics strongly affected by doping using diethylzinc," Nano Lett., vol. 17, no. 2, pp. 702-707, 2017.

[24] J. M. Bao et al., "Optical properties of rotationally twinned InP NW heterostructures," Nano Lett., vol. 8, no. 3, pp. 836-841, 2008.

[25] K. Pemasiri et al., "Carrier dynamics and quantum confinement in type II ZB-WZ InP NW homostructures," Nano Lett., vol. 9, no. 2, pp. 648-654, 2009.

[26] N. Anttu, V. Dagyte, X. Zeng, G. Otnes, and M. Borgstrom, "Absorption and transmission of light in III-V NW arrays for tandem solar cell applications," Nanotechnology, vol. 28, no. 20, 2017, Art. no. 205203.

[27] F. F. Yang et al., "Zn-doping of GaAs NWs grown by Aerotaxy," J. Cryst. Growth, vol. 414, pp. 181-186, 2015.

[28] W. Metaferia et al., "n-Type doping of Aerotaxy-grown GaAs NWs," 2017, in preparation.

[29] E. Barrigón et al., "Aerotaxy GaAs NW pn-junctions," 2017, in preparation.

[30] W. Metaferia et al., "GaAsP NWs grown by Aerotaxy," Nano Lett., vol. 16, no. 9, pp. 5701-5707, 2016.

[31] R. E. Algra et al., "Twinning superlattices in indium phosphide NWs," Nature, vol. 456, no. 7220, pp. 369-372, 2008.

[32] J. Wallentin et al., "Changes in contact angle of seed particle correlated with increased zincblende formation in doped InP NWs," Nano Lett., vol. 10, no. 12, pp. 4807-4812, 2010.

Magnus T. Borgström is an expert in semiconductor epitaxy and nanowire synthesis for electro-optical physics. He is the coordinator of Nanoenergy research, NanoLund, Lund, Sweden ( $h$-index 35 ).

Martin H. Magnusson is leading research in aerosol-based methods to create nanoparticles and nanowires directly in the gas phase, a process known as Aerotaxy ( $h$-index 24).

Frank Dimroth (M'13) is the Head of the Department "III-V Epitaxy and Solar Cells," Fraunhofer Institute for Solar Energy Systems, Freiburg, Germany, and he has 20 years of expertise in high-efficiency III-V multijunction solar cell research ( $h$-index 27).

Gerald Siefer is the head of the team "III-V Cell and Module Characterization" and is a specialist for multijunction solar cell measurement and calibration ( $h$ index 17). 
Oliver Höhn is a project manager with Fraunhofer ISE, Freiburg, Germany, with research focus on optical characterization and optical modeling of highly efficient multijunction solar cells ( $h$-index 7).

Heike Riel (SM'11) is an IBM Fellow, an Executive Director of the IBM Research Frontiers Institute, Ruschlikon, Switzerland, and the Director IoT Technology and Solutions. Her research interests include nanoelectronic and optoelectronic materials and devices ( $h$-index 34$)$.

Heinz Schmid (M'10) is a Senior Engineer with research interests including epitaxy, device fabrication, and electronic and optical devices ( $h$-index 45).

Stephan Wirths is a Post-Doctoral Researcher at IBM, Ruschlikon, Switzerland. His research interests include semiconductor nanowire and layer growth techniques as well as nano- and optoelectronic devices ( $h$-index 15).

Mikael Björk is a CTO with Sol Voltaics AB, Lund, Sweden. Prior to joining the company, he was a permanent research staff member with IBM Research, Switzerland, for six years. He holds several patents with many highly cited scientific papers ( $h$-index 31$)$

Ingvar Åberg (SM'17) received the Ph.D. degree from the Massachusetts Institute of Technology, Cambridge, MA, USA, in 2006.

He is the Director of Cell Engineering with Sol Voltaics AB, Lund, Sweden, leading the company's program on high-efficiency photovoltaic cells based on nanowires ( $h$-index 13 ).
Willie Peijnenburg is a Professor in "environmental toxicology and biodiversity," focusing on the integration of emerging environmental issues and (eco)toxicology ( $h$-index 47).

Martina Vijver is an Assistant Professor with Leiden University, Leiden, The Netherlands. Her research interests include environmental risk assessment and field validation of actual impacts of chemical stressors ( $h$-index 23 ).

Maria Tchernycheva received the Ph.D. degree in physics from the University Paris Sud, Orsay, France, in 2005.

She is currently a CNRS Scientist with 9 years of experience in nanowire physics and applications in charge of NanoPhotoNit Group of IEF ( $h$-index 27).

Valerio Piazza is an early-stage researcher working toward the Ph.D. degree with focus on nanoscale characterizations and nanowire photovoltaic applications ( $h$-index 1$)$

Lars Samuelson (M'17) is recognized for his research on low-dimensiona structures for basic science and applications. He has authored or co-authored more than 600 papers and is the Founder and CSO of Glo AB, Sol Voltaics AB, and Qunano AB, Lund, Sweden ( $h$-index 80). 